\title{
Virtuell und trotzdem ganz AGENS
}

\begin{abstract}
Enno Swart spannte den Boden des ersten virtuellen AGENS Methodenworkshops (AGENSMWS2021v), der am 24. März 2021 stattfand. Zu Beginn brachte er die wohl von vielen mittlerweile gefühlte Frustration mit virtuellen Veranstaltungsformaten zum Ausdruck und konnte am Ende aber dennoch auf einen Workshop zurückblicken, der ganz AGENS war: vielfältig, anspruchsvoll und vor allen Dingen kommunikativ und interaktiv.
\end{abstract}

Der AGENSMWS2021v in Zahlen: maximal 129 Teilnehmer:innen hörten 19 Vorträge in 2 Plenarsitzungen und 4 Break-out-Sessions, begleitet von einem Graphic Recorder, der die Essenz des Tages in einem bunten Tableau festhielt.

Den thematischen Auftakt machte Stefanie March mit ihrem Plenumsvortrag zur Guten Praxis Datenlinkage (GPD), die eines der wesentlichen Ergebnisse der AGENS-Arbeit in den letzten beiden Jahren darstellt und hoffentlich dazu beiträgt, dass dieses wichtige Arbeitsfeld in strukturierten Bahnen läuft. Im Anschluss an das Plenum folgten 4 Break-out-Sessions mit unterschiedlichen thematischen Schwerpunkten:

- Versorgungsforschung

- PKV-Daten und Datenlinkage

- spezielle Methoden

- Populationsbestimmung und ambulante Daten.

Die Bandbreite der eingereichten Abstracts war wie immer groß und reichte von R-Packages zur Aufbereitung von ICD-10-Diagnosen über die Qualitätssicherung von PKV-Daten in der NAKO bis hin zum Abschlussbericht des RADAR-Projektes. Den Abschluss des Tages brachte Stefan Heß vom BfArM mit einem Vortrag zum aktuellen Stand des Forschungsdatenzentrums und einer Umfrage unter den Workshop- 
teilnehmer:innen, in der es um verschiedene Aspekte der zukünftigen Ausgestaltung des Zentrums ging. Die rege Diskussion im Anschluss an diesen Vortrag zeigte einmal mehr, welches Potential das FDZ hat, aber auch, wie hoch die Erwartungshaltung seitens der Community ist.

Es war nicht nur das virtuelle Veranstaltungsformat, das allen Teilnehmer:innen klarmachte, wie außergewöhnlich die Situation angesichts der globalen Corona-Pandemie war und ist. Auf Anregung von Holger Gothe beteiligte sich der AGENSMWS2021v um 12:00 Uhr mittags an der Schweigeminute für die Opfer der Pandemie, zu der das europäische Städtenetzwerk Eurocities aufgerufen hatte. Mit diesem Gedenken ging vermutlich in den Köpfen der meisten der Wunsch einher, dass mithilfe von Impfungen und Schutzmaßnahmen die gegenwärtige Krise bald überwunden werden kann.

Die AGENS richtet in dieser Hinsicht in jedem Fall den Blick nach vorne: der AGENS
Methodenworkshop 2022 wird dankenswerterweise vom Institut für Allgemeinmedizin an der Universität Frankfurt ausgerichtet und soll am 24. und 25. Februar 2022 in der Mainmetropole stattfinden.

Als Vertreter des diesjährigen Gastgebers möchte ich mich ganz herzlich bei allen Teilnehmer:innen und insbesondere bei den Vortragenden bedanken, dass sie diesen Workshop zu dem gemacht haben, was er war. Außerdem gilt mein ganz besonderer Dank meinen Kolleg:innen von der PMV forschungsgruppe, die den Workshop mit viel persönlichem Einsatz vorbereitet und durchgeführt haben.

Zur Nachlese finden sich das Programm, die Abstracts und das Tableau des Graphic Recorders auf der AGENS Homepage unter https://agens.group/.

Ingo Meyer, PMV forschungsgruppe, Köln 